\title{
Design of network video transmission system based on LabVIEW Fuhai HUANG ${ }^{1, a}$, Zhenhai $\mathrm{Wang}^{1}, \mathrm{Xu} \mathrm{Bo}^{2}$ \\ ${ }^{1}$ School of Informatics, Linyi Normal University, Linyi, 276000, China \\ ${ }^{2}$ School of Automobile Engineering, Linyi Normal University, Linyi, 276000, China \\ aemail: hfh8130@163.com
}

Keywords: Virtual Instrument; TCP/IP; Network Transmission

\begin{abstract}
This paper uses LabVIEW software to realize the network online video transmission, introduced the use of TCP/IP protocol on the platform of the virtual instrument. The image collected by the camera on the server through the network transmission to the client, remote video monitoring, at the same time with video storage and other functions.
\end{abstract}

\section{Introduction}

At present, the remote video transmission has been widely used in the fields of education, health and security. Two of the most prominent feature of the video monitoring system is the digital and network. With the progress of society and development of science and technology, the network has spread to thousands of households, has become an indispensable work of learning and living conditions. The virtual instrument is based on the general purpose computer as the core hardware platform, defined by the user design, has a virtual panel, a computer instrument system is carried out by testing software. NI Company's LabVIEW software is the core of software instead of hardware circuits, and simplify the system development, reduce development costs. In this paper, the use of network transmission module of TCP/IP protocol and LabVIEW, puts forward a scheme of the video monitoring system has the characteristics of cheap, practical, universal and stable.

\section{The system design}

This system adopts C/S mode, the client is responsible for a network connection to the server and video monitoring request, receiving server transmits the video, and in the local real-time display and preservation; the server is mainly responsible for the response to the request of the client, the video acquisition equipment to collect the real-time image, and the image information transmission network transmission protocol. Therefore, to realize the remote control of video images through the Internet and related protocol. The structure of the scheme is shown in figure 1.

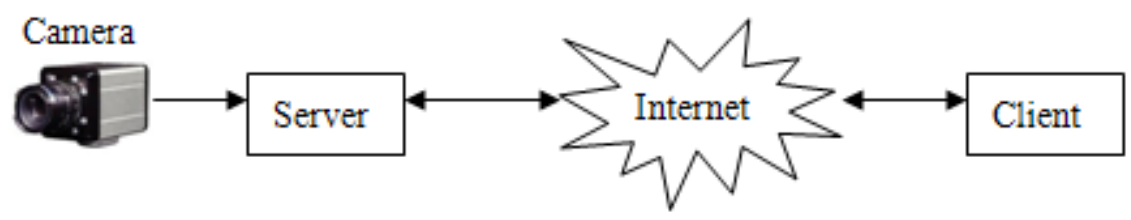

Fig.1. The structure of C/S system

\section{Software design}

Graphical virtual instrument integrated development environment LabVIEW (Laboratory Virtual Instrument Engineering Workbench) is a software development platform of virtual instrument NI company, using the graphical programming language, the program block diagram form, easy to learn and easy to use, can support and expression analysis to source level device its intuitive and easy programming, many the driver, a variety of, have created the basis of conditions for users to quickly and easily build instrument system their own needs in practical production. Compared with the LabVIEW and other computer languages, has a particularly important difference: the other computer languages are used to generate code for text based on LabVIEW language, using the 
graphical programming language.

LabVIEW software provides a visual and motor module, this module can initialize camera and video image capture, as shown in figure 2(a). In addition the TCP protocol controls provide the data communication module, protocol, can be very convenient to establish a network connection and data transmission, as shown in figure 2(b).

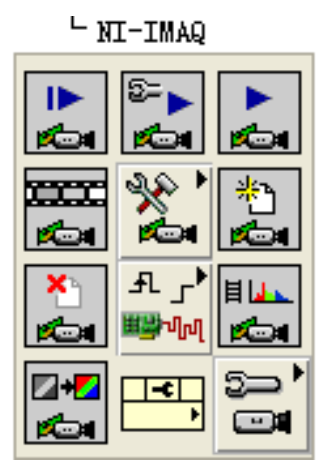

(a) NI-IMAQ module

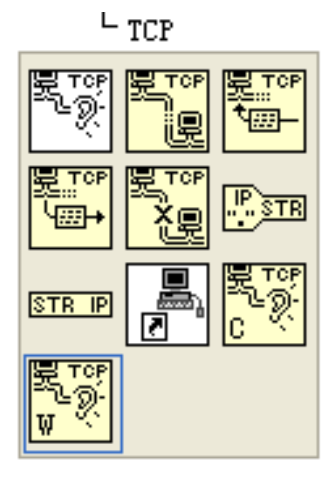

(b) TCP module

Fig.2. LabVIEW module

The system initialization of the camera on the server side, and connected through the TCP protocol to establish with the client, the client to receive and display the video image of the program flow diagram as shown in figure 3.

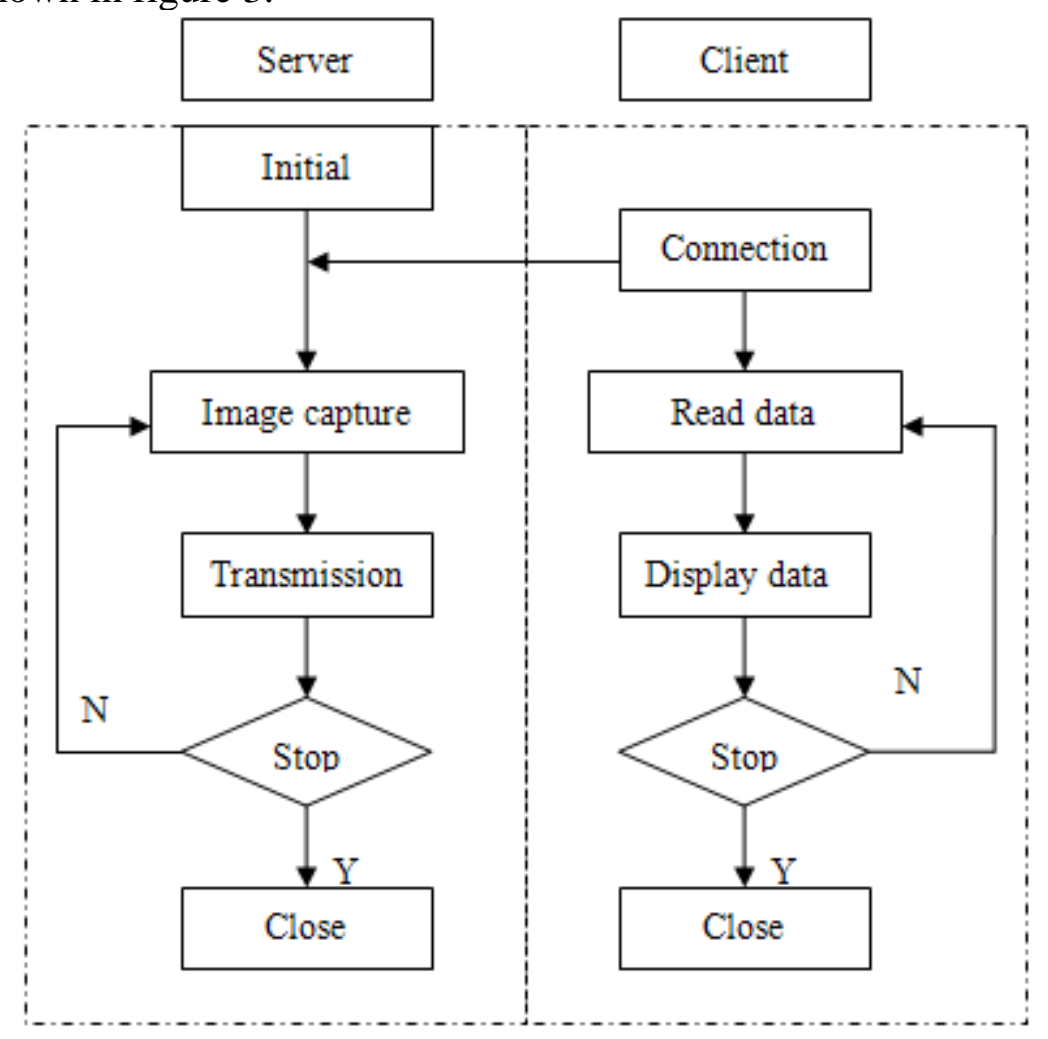

Fig.3. System flow diagram

\section{Server design}

Server side call camera image acquisition. LabVIEW image acquisition is mainly composed of two kinds of methods, one is the use of industrial camera driver, such as LabVIEW driver, DLL dynamic link library; another is the use of NI IMAQ and IMAQdx driver for image acquisition. This design choice is driven by the use of NI for image acquisition IMAQdx. Video image should 
be format conversion before transmission effectively. This design uses the TCP/IP protocol for the video transmission. TCP is a connection based protocol, the transmission must be created before the data transmission. To create a TCP connection, need to specify the address and port of the address. Different ports address different services on the site. Through the "TCP VI Listener" create a listener and wait for a specified port has been received in the TCP connection. If the connection is successful, VI will return a connection handle, connecting the remote TCP address and port of the client. With two 'TCP Write' VI to send data, the first node to transmit data length, the second nodes transmit video data. Finally, the end connected with the TCP Close Connection 'VI'. The server program block diagram shown in figure 4.

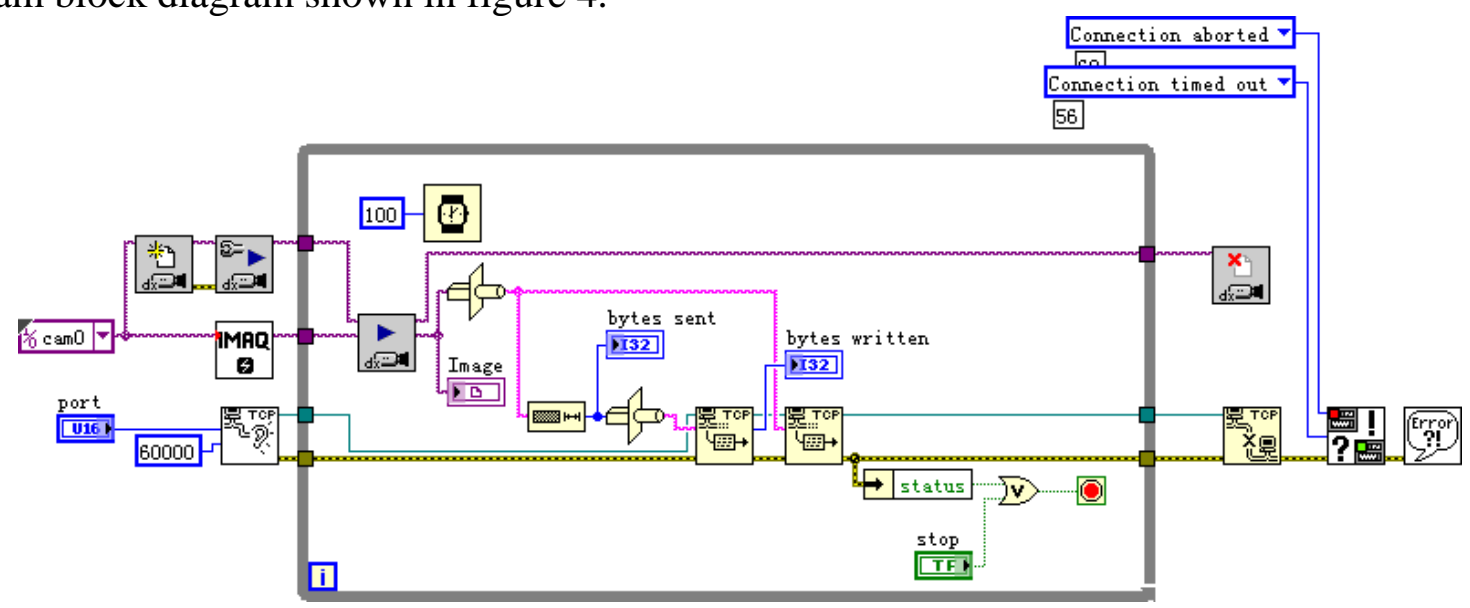

Fig.4. The server program block diagram

\section{The design of the client}

The client is mainly to complete the network connection, read the video data and save functions. 'TCP Open Connection' with VI open TCP, and then use the two 'TCP Read' VI to read the data, the first to receive video data as input length of second nodes, second nodes receive video data, finally, 'TCP Close Connection' by VI end connection. Block diagram as shown in figure 5.

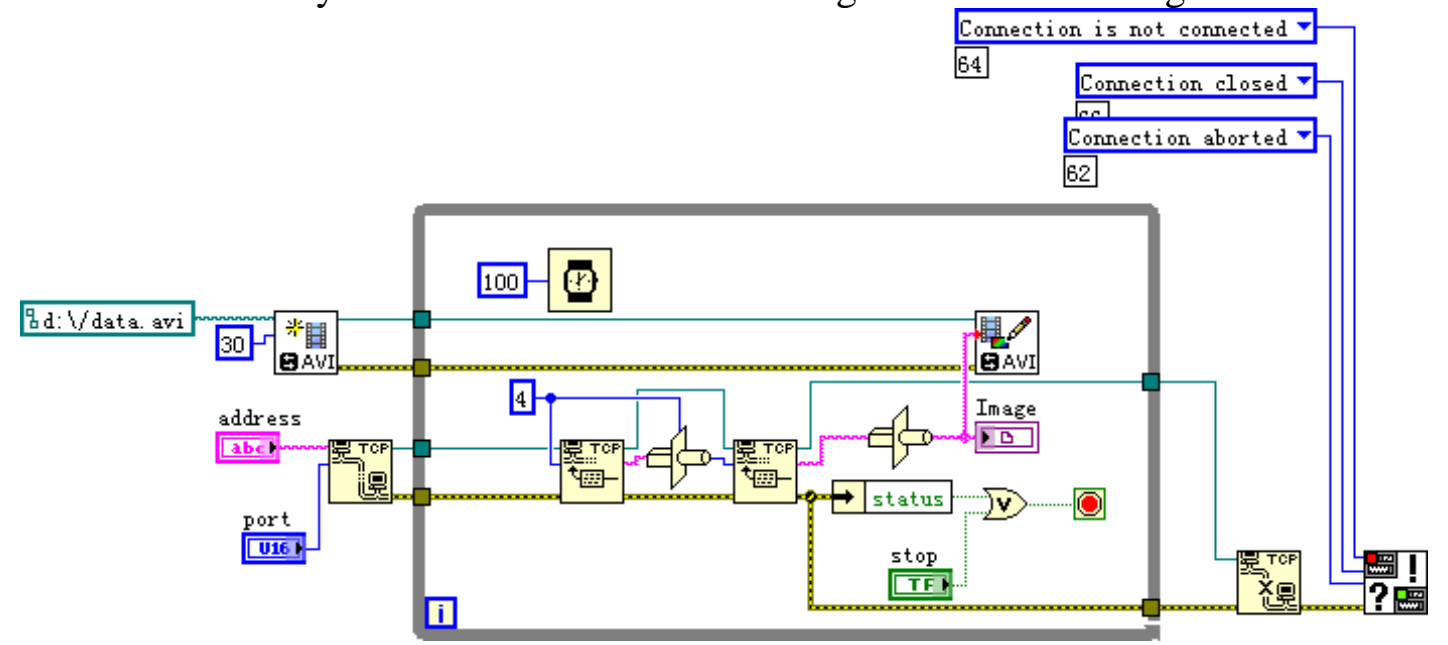

Fig.5. The client program

\section{Test result}

First select a port before the program runs, usually more than 1000, because a lot of less than 1000 of the port is other procedures have been occupied port. Set the server and the client port must be consistent, in order to ensure the normal listening. When the client sends a request to the server in response to the request, the connection, the connection is established, the server will send data package, finally, the client receives the data, and the data unpack restore. The result of running the program is shown in figure 6. 


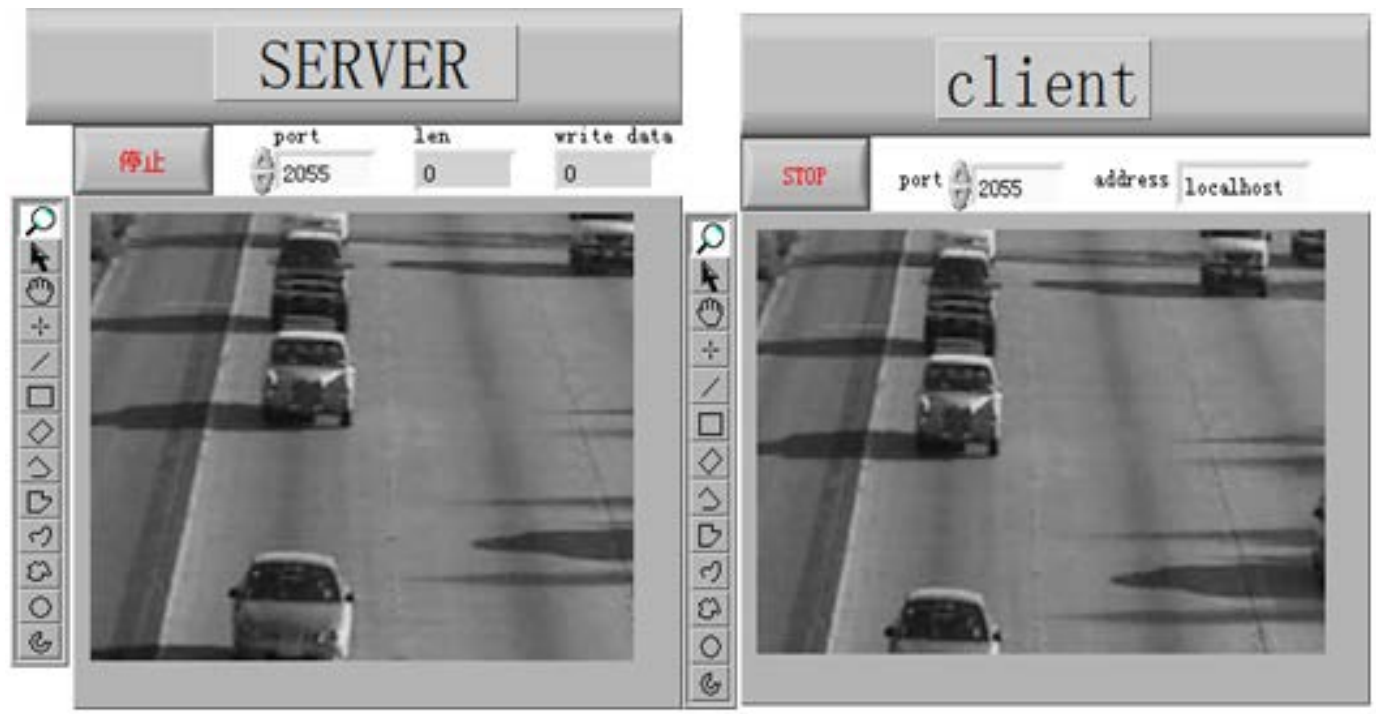

Fig.6. The result of running the program

\section{Conclusion}

This design adopts C/S mode, using LabVIEW platform provides a powerful network functions and network conditions, and combined with the TCP/IP protocol, the effective realization of video network transmission and preservation, by running results show the good performance.

\section{References}

[1]LabVIEW User Manual: USA: National Instruments Corporation (1998).

[2]Changqing Cai, WeiJun Zhang: The design on the multi-temperature testing system based on the Laview, International Seminar on Future Biomedical Information Engineering (FBIE) (2008).

[3]Using External Code in LabVIEW, National Instruments Corporation (2003).

[4]Wagner. C, Armenta. S, Lendl. B: Talanta 80(3)(2010).

[5]F. Correa Alegria, E. Martinho, F. Almeida: Measurement 42(7) (2009). 